\title{
SECOND ORDER ELLIPTIC BOUNDARY VALUE PROBLEMS IN SPACES WITH HOMOGENEOUS NORMS
}

\author{
A. J. PRYDE \\ (Received 9 February 1978) \\ Communicated by Gavin Brown
}

\begin{abstract}
We consider the interior and Dirichlet problems and problems with first order boundary conditions, for a second order homogeneous elliptic partial differential operator with constant coefficients. Under natural conditions on the operators, these problems give rise to isomorphisms between the appropriate spaces with homogeneous norms. From there we obtain a priori estimates and regularity results for boundary value problems in Sobolev-spaces.
\end{abstract}

1980 Mathematics subject classification (Amer. Math. Soc.): 35 J 25.

\section{Introduction}

In Pryde (1979a) we developed the theory of spaces with homogeneous norms. These spaces were an invaluable tool in the study of elliptic partial differential equations with mixed boundary conditions in Sobolev spaces. See Pryde (1979a, 1979b). In those papers, certain of the known results in Sobolev spaces for the interior and Dirichlet problems and those with first order boundary conditions were converted to analogous results in spaces with homogeneous norms. These results were in turn used to consider the mixed problem. However, it is more natural to do things in the reverse order-to prove results in spaces with homogeneous norms, and then convert them to results in Sobolev spaces.

In place of the usual a priori and dual estimates for boundary value problems in Sobolev spaces on $\mathbf{R}_{+}^{n}$ we obtain directly, and fairly simply, isomorphisms between spaces with homogeneous norms. We also show how the relevant conditions on the operators are both necessary and sufficient. The estimates in Sobolev spaces can then be readily obtained, as can regularity results.

(C) Copyright Australian Mathematical Society 1980

Copyright. Apart from any fair dealing for scholarly purposes as permitted under the Copyright Act, no part of this JOURNAL may be reproduced by any process without written permission from the Treasurer of the Australian Mathematical Society. 
For the notation and definitions of the terms used in this paper, the reader should refer to Pryde (1979a, 1979b). We summarize here the definitions of the spaces with homogeneous norms. For $s \geqslant 0, Z^{s}\left(\mathbf{R}^{n}\right)$ is the completion of $C_{0}^{\infty}\left(\mathbf{R}^{n}\right)$ with respect to the norm $\left[u ; \mathbf{R}^{n}\right]_{s}=\left(\int|\xi|^{2 s}|\hat{u}(\xi)|^{2} d \xi\right)^{1 / 2} ; Z^{s}\left(\mathbf{R}_{ \pm}^{n}\right)$ is the closure of $C_{0}^{\infty}\left(\mathbf{R}_{ \pm}^{n}\right)$ in $Z^{s}\left(\mathbf{R}^{n}\right)$; and $Z^{s}\left(\mathbf{R}_{ \pm}^{n}\right)$ is the quotient space $Z^{s}\left(\mathbf{R}^{n}\right) / \dot{Z}^{s}\left(\mathbf{R}_{\mp}^{n}\right)$. For $s<0, Z^{s}\left(\mathbf{R}^{n}\right)$ is the dual of $Z^{-s}\left(\mathbf{R}^{n}\right) ; Z^{s}\left(\mathbf{R}_{ \pm}^{n}\right)$ is the dual of $Z^{-s}\left(\mathbf{R}_{ \pm}^{n}\right)$; and $Z^{s}\left(\mathbf{R}_{ \pm}^{n}\right)$ is the dual of $Z^{-s}\left(\mathbf{R}_{ \pm}^{n}\right)$.

\section{The interior problem}

Let $A=A(D)=\sum_{|\alpha|=2} a_{\alpha} D^{\alpha}$ be a second order homogeneous partial differential operator with constant coefficients. In spaces with homogeneous norms, the interior results take the following simple form.

THEOREM 1.1. For each real s the following are equivalent.

(a) $A: Z^{s}\left(\mathbf{R}^{n}\right) \rightarrow Z^{s-2}\left(\mathbf{R}^{n}\right)$ is an isomorphism.

(b) $A$ is elliptic.

(c) $A: Z^{s}\left(\mathbf{R}^{n}\right) \rightarrow Z^{s-2}\left(\mathbf{R}^{n}\right)$ is left invertible.

Proof. The proof is an immediate consequence of the following lemma.

LEMMA 1.2. Let $P(\xi)$ be a positively homogeneous function on $\mathbf{R}^{n}$ of order $m$ with constant coefficients and continuous for $\xi \neq 0$. Let $P: Z^{s}\left(\mathbf{R}^{n}\right) \rightarrow Z^{s-m}\left(\mathbf{R}^{n}\right)$ be the pseudo-differential operator with symbol $P(\xi)$. The following are equivalent.

(a) $P$ is an isomorphism.

(b) $P(\xi) \neq 0$ for $\xi \neq 0$.

(c) $P$ is left invertible.

Proof. Since $|\nabla|^{s}: Z^{s}\left(\mathbf{R}^{n}\right) \rightarrow L^{2}\left(\mathbf{R}^{n}\right)$ is an isomorphism for all real $s$ (Pryde (1979a)) $P$ is an isomorphism if and only if $Q=|\nabla|^{s-m} P|\nabla|^{-s}: L^{2}\left(\mathbf{R}^{n}\right) \rightarrow L^{2}\left(\mathbf{R}^{n}\right)$ is an isomorphism. But $Q$ has symbol $Q(\xi)=|\xi|^{-m} P(\xi)$ which is positively homogeneous of order 0 and continuous for $\xi \neq 0$.

If $P(\xi) \neq 0$ for $\xi \neq 0, Q(\xi)$ is bounded away from 0 and from $\infty$. So $Q$ is an isomorphism. Hence $(b) \Rightarrow(a) \Rightarrow$ (c).

If $P(\eta)=0$ for some $\eta \in \mathbf{R}^{n}, \eta \neq 0$, consider the functions $u_{\varepsilon} \in L^{2}\left(\mathbf{R}^{n}\right)$, for $\varepsilon>0$, defined by $u_{\varepsilon}(\xi)=\psi((\xi-\eta) / \varepsilon) \varepsilon^{-n / 2}$ where $\psi \in C_{0}^{\infty}\left(\mathbf{R}^{n}\right), \psi=0$ for $|\xi|>1$ and $\|\psi\|_{L^{2}\left(\mathbf{R}^{n}\right)}=1$. Then $\left\|u_{\varepsilon}\right\|_{L^{2}\left(\mathbf{R}^{n}\right)}=1$ and $\left\|Q u_{\epsilon}\right\|_{L^{2}\left(\mathbf{R}^{n}\right)} \leqslant \sup _{|\xi-\eta|<\varepsilon}|Q(\xi)| \rightarrow 0$ as $\varepsilon \rightarrow 0$. So $Q$ is not left invertible and it follows that (c) $\Rightarrow(b)$.

CoRollary 1.3. Let $A$ be elliptic. For each real $s, A: \check{Z}^{s}\left(\mathbf{R}^{n}\right) \rightarrow \dot{Z}^{s-2}\left(\mathbf{R}_{+}^{n}\right)$ is left invertible. 
Proof. $\check{Z}^{s}\left(\mathbf{R}_{+}^{n}\right)$ is a closed subspace of $Z^{s}\left(\mathbf{R}^{n}\right)$.

CoRollaRY 1.4. Let $A$ be elliptic. For each real $s, A: Z^{s}\left(\mathbf{R}_{+}^{n}\right) \rightarrow Z^{s-2}\left(\mathbf{R}_{+}^{n}\right)$ is right invertible.

ProOf. Apply Corollary 1.3 to $A^{\prime}=A^{\prime}(D)=\sum_{|\alpha|=2} \bar{a}_{\alpha} D^{\alpha}$, the formal adjoint of $A$. Then $A^{\prime}: \check{Z}^{2-s}\left(\mathbf{R}_{+}^{n}\right) \rightarrow \check{Z}^{-s}\left(\mathbf{R}_{+}^{n}\right)$ is left invertible. So $\left(A^{\prime}\right)^{*}: Z^{s}\left(\mathbf{R}_{+}^{n}\right) \rightarrow Z^{s-2}\left(\mathbf{R}_{+}^{n}\right)$ is right invertible. As $\left(A^{\prime}\right)^{*}=A$, the corollary is proved.

\section{The Dirichlet problem}

In Pryde (1979a, 1979b) we showed how the trace map $\gamma$ extends to a bounded operator on $Z^{s}\left(\mathbf{R}_{+}^{n}\right)$ for $s>\frac{1}{2}$ and on $Z_{\text {ker }}^{s}\left(\mathbf{R}_{+}^{n}\right)$ for $s<\frac{1}{2}, \not \equiv \frac{1}{2}(\bmod 1)$, provided $A$ is elliptic. For the Dirichlet problem we have the following result.

THEOREM 2.1. If $A$ is elliptic, and $s \neq \frac{1}{2},-\frac{1}{2},-\frac{3}{2}, \ldots$, the following are equivalent

(a) $\gamma: Z_{\mathrm{ker} A}^{s}\left(\mathbf{R}_{+}^{n}\right) \rightarrow Z^{s-\frac{1}{2}}\left(\mathbf{R}^{n-1}\right)$ is an isomorphism.

(b) $A$ is properly elliptic.

(c) $\gamma$ is left invertible.

Proof. Suppose $A$ is properly elliptic. We construct an inverse $E$ of $\gamma$ as follows. Let $m$ be a suitably large integer and $h\left(\xi^{\prime}\right)$, a positively homogeneous function of $\xi^{\prime}=\left(\xi_{1}, \ldots, \xi_{n-1}\right)$ of order 0 , to be determined. For $g \in C_{0}^{\infty}\left(\mathbf{R}^{n-1}\right) \cap Z^{s-\frac{1}{2}}\left(\mathbf{R}^{n-1}\right)$, which is dense in $Z^{s-\frac{1}{1}}\left(\mathbf{R}^{n-1}\right)$, define $Q g$ by

$$
(Q g)^{\wedge}(\xi)=h\left(\xi^{\prime}\right)\left|\xi^{\prime}\right|^{m}\left(\xi_{n}+i\left|\xi^{\prime}\right|\right)^{1-m} A(\xi)^{-1} \hat{g}\left(\xi^{\prime}\right) .
$$

(More precisely, $Q g=|\nabla|^{-8} f$ where $f$ is the $L^{2}$-function with Fourier transform $|\xi|^{s}(Q g)^{\wedge}(\xi)$.) Then $Q$ extends by continuity to a bounded operator

$$
Q: Z^{s-1}\left(\mathbf{R}^{n-1}\right) \rightarrow Z_{\text {ker } R A}^{s}\left(\mathbf{R}^{n}\right) .
$$

(Here, as elsewhere, $R: Z^{s-2}\left(\mathbf{R}^{n}\right) \rightarrow Z^{s-2}\left(\mathbf{R}_{+}^{n}\right)$ is the natural projection.)

Indeed,

$$
\begin{aligned}
\|Q g\|_{Z^{\prime}\left(\mathbf{R}^{n}\right)} \sim\left\||\xi|^{s}(Q g)^{\wedge}(\xi)\right\|_{L^{2}\left(\mathbf{R}^{n)}\right.} \\
\\
\sim\left\|\left|\xi^{\prime}\right|^{m}|\xi|^{s-m-1} \hat{g}\left(\xi^{\prime}\right)\right\|_{L^{2}\left(\mathbf{R}^{n}\right)} \\
\sim\left\|\left|\xi^{\prime}\right|^{-\frac{1}{g}} \hat{g}\left(\xi^{\prime}\right)\right\|_{L^{2}\left(\mathbf{R}^{n-1}\right)}
\end{aligned}
$$


if $m>s-\frac{1}{2}$, because, for $\xi^{\prime} \neq 0$,

where

$$
\begin{aligned}
\int_{-\infty}^{\infty}\left|\xi^{\prime}\right|^{2 m}|\xi|^{2 s-2 m-2} d \xi_{n} & =\int_{-\infty}^{\infty}\left|\xi^{\prime}\right|^{2 m}\left(\xi_{n}^{2}+\left|\xi^{\prime}\right|^{2}\right)^{s-m-1} d \xi_{n} \\
& =\left|\xi^{\prime}\right|^{2 s-1} \int_{-\infty}^{\infty}\left(1+t^{2}\right)^{s-m-1} d t \\
& =K\left|\xi^{\prime}\right|^{2 s-1}
\end{aligned}
$$

$$
K=\int_{-\infty}^{\infty}\left(1+t^{2}\right)^{s-m-1} d t<\infty
$$

So $\|Q g\|_{Z^{x}\left(\mathbf{R}^{n}\right)} \sim\|g\|_{Z^{-\frac{1}{2}\left(\mathbf{R}^{n-1}\right)}}$.

Further,

$$
w=\left(D_{n}+i\left|\nabla^{\prime}\right|\right)^{s-2} A Q g \in L^{2}\left(\mathbf{R}^{n}\right)
$$

and

$$
\hat{w}(\xi)=h\left(\xi^{\prime}\right)\left|\xi^{\prime}\right|^{m}\left(\xi_{n}+i\left|\xi^{\prime}\right|\right)^{s-m-1} \hat{g}\left(\xi^{\prime}\right)
$$

So (1) $\hat{w}\left(\xi^{\prime}, \xi_{n}\right)$ has an analytic extension to $\operatorname{Im} \xi_{n}>0$ and (2)

$$
\sup _{\eta>0}\left\|\hat{w}\left(\xi^{\prime}, \xi_{n}+i \eta\right)\right\|_{L^{2}\left(\mathbf{R}^{n)}\right.}=\left\|\hat{w}\left(\xi^{\prime}, \xi_{n}\right)\right\|_{L^{2}\left(\mathbf{R}^{n}\right)}<\infty
$$

Hence $w \in L^{2}\left(\mathbf{R}_{-}^{n}\right)$. But $\left.\left(D_{n}+i\left|\nabla^{\prime}\right|\right)^{s-2}: \stackrel{\circ}{Z}^{s-2} \mathbf{R}_{-}^{n}\right) \rightarrow L^{2}\left(\mathbf{R}_{-}^{n}\right)$ is an isomorphism (Pryde (1979a)) and so $A Q g \in \dot{Z}^{s-2}\left(\mathbf{R}_{-}^{n}\right)$. In other words, $R A Q g=0$, or $Q g \in Z_{\text {ker } R A}^{s}\left(\mathbf{R}^{n}\right)$. So $Q$ extends by continuity as claimed.

Defining $E=R Q: Z^{s-\frac{1}{2}}\left(\mathbf{R}^{n-1}\right) \rightarrow Z_{\text {ker } A}^{s}\left(\mathbf{R}_{+}^{n}\right)$ it remains to show that $\gamma E=I$ and $E \gamma=I$.

For $g \in C_{0}^{\infty}\left(\mathbf{R}^{n-1}\right) \cap Z^{s-\frac{1}{2}}\left(\mathbf{R}^{n-1}\right)$, and $\xi^{\prime} \neq 0$,

$$
\begin{aligned}
(\gamma E g)^{\wedge}\left(\xi^{\prime}\right) & =(\gamma Q g)^{\wedge}\left(\xi^{\prime}\right) \\
& =(2 \pi)^{-\frac{1}{2}} \int_{-\infty}^{\infty}(Q g)^{\wedge}\left(\xi^{\prime}, \xi_{n}\right) d \xi_{n} \\
& =(2 \pi)^{-1} \int_{-\infty}^{\infty} h\left(\xi^{\prime}\right)\left|\xi^{\prime}\right|^{m}\left(\xi_{n}+i\left|\xi^{\prime}\right|\right)^{1-m} A\left(\xi^{\prime}, \xi_{n}\right)^{-1} \hat{g}\left(\xi^{\prime}\right) d \xi_{n} .
\end{aligned}
$$

Moreover, since $A$ is properly elliptic, $A\left(\xi^{\prime}, \xi_{n}\right)=a_{0}\left(\xi_{n}-\tau^{+}\left(\xi^{\prime}\right)\right)\left(\xi_{n}-\tau^{-}\left(\xi^{\prime}\right)\right)$, where $\operatorname{Im} \tau^{+}\left(\xi^{\prime}\right)>0$ and $\operatorname{Im} \tau^{-}\left(\xi^{\prime}\right)<0$, for $\xi^{\prime} \neq 0$. So the integrand is analytic in the 
upper half complex $\xi_{n}$-plane apart from one first order pole at $\xi_{n}=\tau^{+}\left(\xi^{\prime}\right)$. If $m>0$,

$$
\begin{aligned}
(\gamma E g)^{\wedge}\left(\xi^{\prime}\right) & =(2 \pi i) \cdot\left(\text { residue of }(2 \pi)^{-\frac{1}{2}}(Q g)^{\wedge}\left(\xi^{\prime}, \xi_{n}\right) \text { at } \tau^{+}\left(\xi^{\prime}\right)\right) \\
& =(2 \pi i)(2 \pi)^{-\frac{1}{2}} h\left(\xi^{\prime}\right)\left|\xi^{\prime}\right|^{m}\left(\tau^{+}\left(\xi^{\prime}\right)+i\left|\xi^{\prime}\right|\right)^{1-m} a_{0}^{-1}\left(\tau^{+}\left(\xi^{\prime}\right)-\tau^{-}\left(\xi^{\prime}\right)\right)^{-1} \hat{g}\left(\xi^{\prime}\right) \\
& =\hat{g}\left(\xi^{\prime}\right),
\end{aligned}
$$

provided $h\left(\xi^{\prime}\right)=(2 \pi i)^{-1}(2 \pi)^{\frac{1}{2}}\left|\xi^{\prime}\right|^{-m}\left(\tau^{+}\left(\xi^{\prime}\right)+i\left|\xi^{\prime}\right|\right)^{m-1} a_{0}\left(\tau^{+}\left(\xi^{\prime}\right)-\tau^{-}\left(\xi^{\prime}\right)\right)$. So $\gamma E=I$ as required.

Finally, we show $E \gamma=I$ on $Z_{\text {ker } A}^{s}\left(\mathbf{R}_{+}^{n}\right)$. Since $H_{\text {ker } A}^{s^{\prime}}\left(\mathbf{R}_{+}^{n}\right)$ is dense in $Z_{\text {ker } A}^{s}\left(\mathbf{R}_{+}^{n}\right)$ for all $s^{\prime}$ (Pryde (1979b), Proposition 4.3) it suffices to take $s \geqslant 2$. Let $u \in Z_{\mathrm{ker} A}^{s}\left(\mathbf{R}_{+}^{n}\right)$. Let $P$ be the reflection operator constructed in Pryde (1979b), Section 4. So $P: H^{m}\left(\mathbf{R}_{+}^{n}\right) \rightarrow H^{m}\left(\mathbf{R}^{n}\right)$ is bounded and satisfies $R P=I$. Moreover, if $0 \leqslant s \leqslant m$, $P: Z^{s}\left(\mathbf{R}_{+}^{n}\right) \rightarrow Z^{s}\left(\mathbf{R}^{n}\right)$ is bounded and $R P=I$. So $E \gamma u=R Q \gamma u=R Q \gamma P u$. Setting $w=\left(D_{n}+i\left|\nabla^{\prime}\right|\right)^{s}(Q \gamma P u-P u) \in L^{2}\left(\mathbf{R}^{n}\right)$, it suffices to prove that $w \in L^{2}\left(\mathbf{R}_{-}^{n}\right)$. For, then, $Q \gamma P u-P u \in \dot{Z}^{s}\left(\mathbf{R}_{-}^{n}\right)$ and $R Q \gamma P u=R P u=u$.

Now $R A P u=0$, so $A P u=v \in \dot{Z}^{s-2}\left(\mathbf{R}_{-}^{n}\right)$. Let $v_{i} \in C_{0}^{\infty}\left(\mathbf{R}_{-}^{n}\right), v_{i} \rightarrow v$ in $\dot{Z}^{s-2}\left(\mathbf{R}_{-}^{n}\right)$. So $R A^{-1} v_{i} \rightarrow u$ in $Z_{\text {ker } A}^{s}\left(\mathbf{R}_{+}^{n}\right)$ and $w_{i}=\left(D_{n}+i\left|\nabla^{\prime}\right|\right)^{s}\left(Q \gamma A^{-1} v_{i}-A^{-1} v_{i}\right) \rightarrow w$ in $L^{2}\left(\mathbf{R}^{n}\right)$. Also

$$
\begin{gathered}
\hat{w}_{i}(\xi)=h\left(\xi^{\prime}\right)\left|\xi^{\prime}\right|^{m}\left(\xi_{n}+i\left|\xi^{\prime}\right|\right)^{s+1-m} A(\xi)^{-1}(2 \pi)^{-\frac{1}{2}} \int_{-\infty}^{\infty} A\left(\xi^{\prime}, t\right)^{-1} \hat{v}_{i}\left(\xi^{\prime}, t\right) d t \\
-\left(\xi_{n}+i\left|\xi^{\prime}\right|\right)^{s} A(\xi)^{-1} \hat{v}_{i}(\xi) .
\end{gathered}
$$

Consider then the positively oriented contour $C_{r}=[-r, r] \cup S_{r}$, where $S_{r}$ is the semi-circle $|z|=r, \operatorname{Im} z \geqslant 0$. For sufficiently large $r$,$$
\int_{C_{r}} A\left(\xi^{\prime}, z\right)^{-1} \hat{v}_{i}\left(\xi^{\prime}, z\right) d z=(2 \pi i) a_{0}^{-1}\left(\tau^{+}\left(\xi^{\prime}\right)-\tau^{-}\left(\xi^{\prime}\right)\right)^{-1} \hat{v}_{i}\left(\xi^{\prime}, \tau^{+}\left(\xi^{\prime}\right)\right) .
$$

But

$$
\begin{aligned}
\left|\int_{S_{r}} A\left(\xi^{\prime}, z\right)^{-1} \hat{v}_{i}\left(\xi^{\prime}, z\right) d z\right| & \leqslant \int_{0}^{\pi}\left|A\left(\xi^{\prime}, r e^{i \theta}\right)\right|^{-1}\left|\hat{v}_{i}\left(\xi^{\prime}, r e^{i \theta}\right)\right| r d \theta \\
& \leqslant \frac{C}{r} \int_{0}^{\pi}\left|\hat{v}_{i}\left(\xi^{\prime}, r e^{i \theta}\right)\right| d \theta
\end{aligned}
$$

for $r$ large enough. But

$$
\left|\hat{v}_{i}\left(\xi^{\prime}, r e^{i \theta}\right)\right|=\left|\int_{-\infty}^{0} e^{-i x_{n} r e^{i \theta}} \tilde{v}_{i}\left(\xi^{\prime}, x_{n}\right) d x_{n}\right|
$$

$\left(\tilde{v}\left(\xi^{\prime}, x_{n}\right)\right.$ denoting the Fourier transform with respect to the first $n-1$ variables 
only)

$$
\leqslant \int_{-\infty}^{0}\left|\tilde{v}_{i}\left(\xi^{\prime}, x_{n}\right)\right| d x_{n}, \quad \text { if } 0 \leqslant \theta \leqslant \pi
$$

(because $\left.\left|\exp \left(-i x_{n} r e^{i \theta}\right)\right|=\left|\exp \left(-i x_{n} r(\cos \theta+i \sin \theta)\right)\right|=\exp \left(r x_{n} \sin \theta\right) \leqslant 1\right)$

$<\infty$, because $v_{i} \in C_{0}^{\infty}\left(\mathbf{R}_{-}^{n}\right)$.

Therefore

$$
\int_{S_{r}} A\left(\xi^{\prime}, z\right)^{-1} \hat{v}_{i}\left(\xi^{\prime}, z\right) d z \rightarrow 0 \text { as } r \rightarrow \infty
$$

and so, for $\xi^{\prime} \neq 0$,

$$
\int_{-\infty}^{\infty} A\left(\xi^{\prime}, t\right)^{-1} \hat{v}_{i}\left(\xi^{\prime}, t\right) d t=(2 \pi i) a_{0}^{-1}\left(\tau^{+}\left(\xi^{\prime}\right)-\tau^{-}\left(\xi^{\prime}\right)\right)^{-1} \hat{v}_{i}\left(\xi^{\prime}, \tau^{+}\left(\xi^{\prime}\right)\right) .
$$

Hence

$$
\begin{aligned}
\hat{w}_{i}(\xi)=\left[\left(\tau^{+}\left(\xi^{\prime}\right)+i\left|\xi^{\prime}\right|\right)^{m-1} \tilde{v}_{i}\left(\xi^{\prime}, \tau^{+}\left(\xi^{\prime}\right)\right)\right. \\
\\
\left.-\left(\xi_{n}+i\left|\xi^{\prime}\right|\right)^{m-1} \hat{v}_{i}(\xi)\right] A(\xi)^{-1}\left(\xi_{n}+i\left|\xi^{\prime}\right|\right)^{s+1-m} .
\end{aligned}
$$

The expression in square brackets and $A(\xi)$ each have a zero at $\xi_{n}=\tau^{+}\left(\xi^{\prime}\right)$. Hence $\hat{w}_{i}\left(\xi^{\prime}, \xi_{n}\right)$ is analytic in $\operatorname{Im} \xi_{n}>0$, for $\xi^{\prime} \neq 0$. Moreover, since

$$
\left(D_{n}+i\left|\nabla^{\prime}\right|\right)^{m-1} v_{i} \in L^{2}\left(\mathbf{R}_{-}^{n}\right),
$$

it follows from Lemma 2.2 below that $w_{i} \in L^{2}\left(\mathbf{R}_{-}^{n}\right)$. Hence $w \in L^{2}\left(\mathbf{R}_{-}^{n}\right)$ ) as required, and so $(\mathrm{b}) \Rightarrow(\mathrm{a}) \Rightarrow$ (c).

To show (c) $\Rightarrow($ b), suppose $A$ is not properly elliptic. Then $n=2$ and $A(\xi)=a_{0}\left(\xi_{2}-\xi_{1} \tau_{1}\right)\left(\xi_{2}-\xi_{1} \tau_{2}\right)$ where $\left(\operatorname{Im} \tau_{1}\right)\left(\operatorname{Im} \tau_{2}\right)>0$. Suppose $\operatorname{Im} \tau_{j}>0$ for $j=1,2$. Let $g \in C_{0}^{\infty}\left(\mathbf{R}_{+}^{1}\right)$ and define $v\left(x_{1}, x_{2}\right)$ by

$$
\begin{aligned}
& \tilde{v}\left(\xi_{1}, x_{2}\right)=g\left(\xi_{1}\right)\left(\exp \left(i \tau_{1} \xi_{1} x_{2}\right)-\exp \left(i \tau_{2} \xi_{1} x_{2}\right)\right) \quad \text { if } \quad \tau_{1} \neq \tau_{2}, \\
& =g\left(\xi_{1}\right) x_{2} \exp \left(i \tau_{1} \xi_{1} x_{2}\right) \quad \text { if } \tau_{1}=\tau_{2} .
\end{aligned}
$$

We show that $v \in \operatorname{ker} \gamma$. Firstly,

$$
\begin{aligned}
\int_{0}^{\infty} \int_{-\infty}^{\infty}\left|\tilde{v}\left(\xi_{1}, x_{2}\right)\right|^{2} d \xi_{1} d x_{2} & \leqslant-\frac{1}{2}\left(\frac{1}{\operatorname{Im} \tau_{1}}+\frac{1}{\operatorname{Im} \tau_{2}}\right) \int_{0}^{\infty} \frac{\left|g\left(\xi_{1}\right)\right|^{2}}{\xi_{1}} d \xi_{1} \\
& <\infty
\end{aligned}
$$

so $v \in L^{2}\left(\mathbf{R}_{+}^{2}\right)$. Similarly, $D^{\alpha} v \in L^{2}\left(\mathbf{R}_{+}^{2}\right)$ for all multi-indices $\alpha$. So $v \in H^{s}\left(\mathbf{R}_{+}^{2}\right)$ for all $s$ and therefore $v \in Z^{s}\left(\mathbf{R}_{+}^{2}\right)$ for all $s \geqslant 0$. As well, $v \in Z^{-s}\left(\mathbf{R}_{+}^{2}\right)$ for all $s \geqslant 0$. Indeed, if 
$\varphi \in C_{0}^{\infty}\left(\mathbf{R}_{+}^{2}\right)$ and $v_{1}$ is $v$ extended arbitrarily to a function in $L^{2}\left(\mathbf{R}^{2}\right)$ then $\langle v, \varphi\rangle=\int_{\mathbf{R}_{+}{ }^{2}} v(x) \overline{\varphi(x)} d x$ satisfies

$$
\begin{aligned}
|\langle v, \varphi\rangle| & =\left|\int_{\mathbf{R}^{2}} v_{1}(x) \overline{\varphi(x)} d x\right| \\
& \leqslant\left\||\xi|^{-s} \hat{v}_{1}\right\|_{L^{2}\left(\mathbf{R}^{2}\right)} \cdot\left\|\left.\xi\right|^{s} \varphi\right\|_{L^{2}\left(\mathbf{R}^{2}\right)} \\
& =c\|\varphi\|_{Z^{*}\left(\mathbf{R}_{+}{ }^{2}\right)} .
\end{aligned}
$$

So $\langle v, \varphi\rangle$ extends by continuity to a bounded form on $\dot{Z}^{s}\left(\mathbf{R}_{+}^{\mathbf{2}}\right)$. Hence $v \in Z^{-s}\left(\mathbf{R}_{+}^{\mathbf{2}}\right)$. Now

$$
\begin{aligned}
(A v)^{\sim}\left(\xi_{1}, x_{2}\right) & =a_{0}\left(D_{2}-\xi_{1} \tau_{1}\right)\left(D_{2}-\xi_{1} \tau_{2}\right) \tilde{v}\left(\xi_{1}, x_{2}\right) \\
& =0 .
\end{aligned}
$$

Hence $v \in Z_{\text {ker } A}^{s}\left(\mathbf{R}_{+}^{2}\right)$. Finally, $(\gamma v)^{\wedge}\left(\xi_{1}\right)=\tilde{v}\left(\xi_{1}, 0\right)=0$ and so $v \in \operatorname{ker} \gamma$. Hence $\gamma$ is not left invertible. So (c) $\Rightarrow(\mathrm{b})$.

Lemma 2.2. If $v \in L^{2}\left(\mathbf{R}_{-}^{\mathbf{1}}\right)$ and $\hat{w}(\xi)=\left(\hat{v}(\xi)-\hat{v}\left(z_{0}\right)\right) /\left(\xi-z_{0}\right)$ then $\hat{w}(\xi)$ is the Fourier transform of a function $w \in L^{2}\left(\mathbf{R}_{-}^{1}\right)$.

Proof. We have to prove

(1) $\hat{w}(\xi)$ has an analytic extension to $\operatorname{Im} \xi>0$.

(2) $\sup _{\eta>0}\|\hat{w}(\xi+i \eta)\|_{L^{2}\left(\mathbf{R}^{1)}\right.}<\infty$.

But (1) follows from the same property for $\hat{v}(\xi)$ and (2) follows similarly, using, near the line $\eta=\operatorname{Im} z_{0}$, the analyticity of $\hat{w}(\xi)$.

COROLlary 2.3. If $A$ is elliptic and $s>\frac{1}{2}$, the following are equivalent.

(a) $(A, \gamma): Z^{s}\left(\mathbf{R}_{+}^{n}\right) \rightarrow Z^{s-2}\left(\mathbf{R}_{+}^{n}\right) \times Z^{s-\frac{1}{2}}\left(\mathbf{R}^{n-1}\right)$ is an isomorphism.

(b) $A$ is properly elliptic.

(c) $(A, \gamma)$ is left invertible.

Proof. By Corollary 1.4, $A: Z^{s}\left(\mathbf{R}_{+}^{n}\right) \rightarrow Z^{s-2}\left(\mathbf{R}_{+}^{n}\right)$ is right invertible. It follows from application 4.2 of the five lemmas of Pryde (1977) that $(A, \gamma)$ is an isomorphism (or left invertible) if and only if $\gamma / \operatorname{ker} A$ is an isomorphism (or left invertible).

\section{Problems with first order boundary conditions}

Suppose now that $b=b(D)=\sum_{j=1}^{n} b_{j} D_{j}$ is a first order homogeneous operator with constant coefficients. Using the trace operator $\gamma$ we obtain a bounded operator $B=\gamma b: Z_{\mathrm{ker} A}^{s}\left(\mathbf{R}_{+}^{n}\right) \rightarrow Z^{s-(3 / 2)}\left(\mathbf{R}^{n-1}\right)$ for $s \neq \frac{3}{2}, \frac{1}{2},-\frac{1}{2}, \ldots$. 
If $A$ is properly elliptic and $B^{\prime}\left(\xi^{\prime}\right)=b\left(\xi^{\prime}, \tau^{+}\left(\xi^{\prime}\right)\right)$ then $B$ satisfies the complementing condition with respect to $A$ on the boundary $\mathbf{R}^{n-1}$ of $\mathbf{R}_{+}^{n}$ if and only if $B^{\prime}\left(\xi^{\prime}\right) \neq 0$ for real $\xi^{\prime} \neq 0$.

The following result is proved in Pryde (1979b), Lemma 6.1.

LEMMA 3.1. If $A$ is properly elliptic and $s \neq \frac{3}{2}, \frac{1}{2},-\frac{1}{2}, \ldots$ then $B u=B^{\prime} \gamma u$ for all $u \in Z_{\text {ker } A}^{s}\left(\mathbf{R}_{+}^{n}\right)$.

For the problems with first order boundary conditions we have the result

THEOREM 3.2. If $A$ is properly elliptic and $s \neq \frac{3}{2}, \frac{1}{2},-\frac{1}{2}, \ldots$ the following are equivalent.

(a) $B: Z_{\text {ker } A}^{s}\left(\mathbf{R}_{+}^{n}\right) \rightarrow Z^{s-(3 / 2)}\left(\mathbf{R}^{n-1}\right)$ is an isomorphism.

(b) $B$ satisfies the complementing condition.

(c) $B$ is left invertible.

Proof. By Lemma 3.1 the following diagram commutes

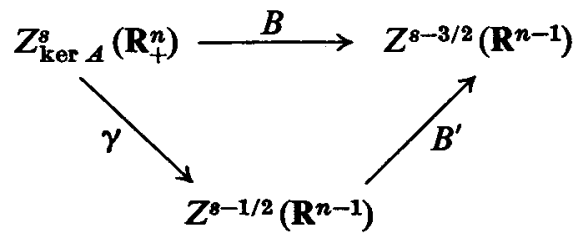

By Theorem 2.1, $\gamma$ is an isomorphism. Hence $B$ is left invertible or an isomorphism if and only if $B^{\prime}$ is left invertible or an isomorphism. But, by Lemma 1.2, each of these last properties is equivalent to the complementing condition holding.

COROLlaRY 3.3. If $A$ is properly elliptic and $s>\frac{3}{2}$ the following are equivalent.

(a) $(A, B): Z^{s}\left(\mathbf{R}_{+}^{n}\right) \rightarrow Z^{s-2}\left(\mathbf{R}_{+}^{n}\right) \times Z^{s-(3 / 2)}\left(\mathbf{R}^{n-1}\right)$ is an isomorphism.

(b) $B$ satisfies the complementing condition.

(c) $(A, B)$ is left invertible.

Proof. The proof is the same as that of Corollary 2.3.

\section{Related results in Sobolev spaces}

Here we use the results of the previous sections to obtain known estimates for various boundary value problems in Sobolev spaces. Similar estimates (for integer s) were originally found by Agmon et al. (1959), Browder (1959) and Schechter (1959). 
Let $A=A(D)=\sum_{|\alpha| \leqslant 2} a_{\alpha} D^{\alpha}$ be a second order operator with constant coefficients and $B=B(D)=\sum_{i=1}^{n} b_{i} D_{i}+b_{0}$ be a first order boundary operator with constant coefficients. Let $Q=\left\{x \in \mathbf{R}^{n}:|x|<1\right\}$ and $Q_{+}=\left\{x \in Q: x_{n}>0\right\}$.

THEOREM 4.1. Let $s$ be real. The estimate

$$
\|u\|_{H^{s}\left(\mathbf{R}^{n)}\right.} \leqslant c\left(\|A u\|_{H^{*-2}\left(\mathbf{R}^{n}\right)}+\|u\|_{H^{*-1}\left(\mathbf{R}^{n}\right)}\right) \text { for all } u \in H^{s}(Q),
$$

holds if and only if $A$ is elliptic.

Proof. We may suppose that $A(\xi)$ is homogeneous of order 2, since lower order terms can be asborbed into the remainder term $\|u\|_{H^{a-1}\left(\mathbf{R}^{n}\right)}$ in estimate (4.1).

Suppose then (4.1) holds. Let $u \in C_{0}^{\infty}\left(\mathbf{R}^{n}\right) \cap Z^{s}\left(\mathbf{R}^{n}\right)$ which is dense in $Z^{s}\left(\mathbf{R}^{n}\right)$. Then $u_{\varepsilon}$ defined by $u_{\varepsilon}(x)=\varepsilon^{s-(n / 2)} u(x / \varepsilon)$ is in $C_{0}^{\infty}(Q)$ provided $0<\varepsilon \leqslant \varepsilon_{0}$, say.

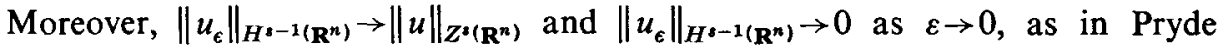
(1979a). So, from (4.1) applied to $u_{\epsilon}$, we obtain

$$
\|u\|_{Z^{s}\left(\mathbf{R}^{n)}\right.} \leqslant c\|A u\|_{Z^{s-2}\left(\mathbf{R}^{n}\right)} \text { for all } u \in C_{0}^{\infty}\left(\mathbf{R}^{n}\right) \cap Z^{s}\left(\mathbf{R}^{n}\right) .
$$

Hence $A$ is left invertible, and, by Theorem 1.1, $A$ is elliptic.

Conversely, suppose $A$ is elliptic. Then in particular $\left(4.1^{\prime}\right)$ holds for all $u \in C_{0}^{\infty}(Q) \cap Z^{s}\left(\mathbf{R}^{n}\right)$. By Pryde (1979a) the Sobolev and homogeneous norms are equivalent on this last space and so

$$
\|u\|_{H^{*}\left(\mathbf{R}^{n}\right)} \leqslant c\|A u\|_{H^{\operatorname{s-2}\left(\mathbf{R}^{n}\right)}} \text { for all } u \in C_{0}^{\infty}(Q) \cap Z^{s}\left(\mathbf{R}^{n}\right) .
$$

But the closure of $C_{0}^{\infty}(Q) \cap Z^{s}\left(\mathbf{R}^{n}\right)$ in $\stackrel{H}{H}^{s}(Q)$ has finite codimension. On any complement of that closure, $\|u\|_{H^{2}\left(R^{n}\right)} \sim\|u\|_{H^{t-1}\left(R^{n}\right)}$ and so estimate (4.1) follows.

In analogous fashion we obtain the following results from Theorems 2.1 and 3.2 and their corollaries.

THeOREM 4.2. Let $A$ be homogeneous and elliptic with $s \neq \frac{1}{2},-\frac{1}{2},-\frac{3}{2}, \ldots$. The estimate

$$
\begin{array}{r}
\|u\|_{H^{\bullet}\left(\mathbf{R}_{+}^{n}\right)} \leqslant c\left(\|\gamma u\|_{H^{s-1 / 2}\left(\mathbf{R}^{n-1)}+\|u\|_{H^{s-1}\left(\mathbf{R}_{+}^{n}\right)}\right)}\right. \\
\text { for all } u \in H_{\mathbf{k e r} A^{s}}^{s}\left(\mathbf{R}_{+}^{n}\right) \text { with support in } \overline{Q_{+}},
\end{array}
$$

holds if and only if $A$ is properly elliptic.

THEOREM 4.3. Let $A$ be elliptic with $s>\frac{1}{2}$. The estimate

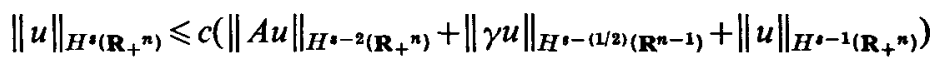

for all $u \in H^{s}\left(\mathbf{R}_{+}^{n}\right)$ with support in $\overline{Q_{+}}$, 
holds if and only if $A$ is properly elliptic.

THEOREM 4.4. Let $A$ be homogeneous and properly elliptic with $s \neq \frac{3}{2}, \frac{1}{2},-\frac{1}{2}, \ldots$. The estimate

$$
\begin{array}{r}
\|u\|_{H^{\prime}\left(\mathbf{R}_{+}^{n}\right)} \leqslant c\left(\|B u\|_{H^{s-(3 / 2)}\left(\mathbf{R}_{+}^{n}\right)}+\|u\|_{H^{s-1}\left(\mathbf{R}_{+}^{n j}\right)}\right) \\
\text { for all } u \in H_{\mathbf{k e r} A^{s}}^{s}\left(\mathbf{R}_{+}^{n}\right) \text { with support in } \overline{Q_{+}},
\end{array}
$$

holds if and only if $B$ satisfies the complementing condition.

THEOREM 4.5. Let $A$ be properly elliptic with $s>\frac{3}{2}$. The estimate

$$
\begin{gathered}
\|u\|_{H^{s}\left(\mathbf{R}_{+}^{n}\right)} \leqslant c\left(\|A u\|_{H^{s-2}\left(\mathbf{R}_{+}^{n}\right)}+\|B u\|_{H^{s-(3 / 2)}\left(\mathbf{R}^{n-1}\right)}+\|u\|_{H^{i-1}\left(\mathbf{R}_{+}^{n}\right)}\right) \\
\text { for all } u \in H^{s}\left(\mathbf{R}_{+}^{n}\right) \text { with support in } \overline{Q_{+}}
\end{gathered}
$$

holds if and only if B satisfies the complementing condition.

\section{Regularity results}

Let $A$ and $B$ be constant coefficient operators, not necessarily homogeneous, as in the previous section.

THEOREM 5.1. Let $A$ be elliptic with $s$ real.

(a) If $A$ is homogeneous, with $u \in L^{2}\left(\mathbf{R}^{n}\right)$ and $A u \in Z^{s-2}\left(\mathbf{R}^{n}\right)$ then $u \in H^{8}\left(\mathbf{R}^{n}\right)$.

(b) In general, if $u \in L^{2}\left(\mathbf{R}^{n}\right)$ and $A u \in H^{s-2}\left(\mathbf{R}^{n}\right)$ then $u \in H^{s}\left(\mathbf{R}^{n}\right)$.

Proof.

(a) $\|u\|_{Z^{s}\left(\mathbf{R}^{n}\right)} \sim\left\||\xi|^{s} \hat{u}\right\|_{L^{2}\left(\mathbf{R}^{n}\right)}$

$$
\sim\left\||\xi|^{s-2}(A u)^{\wedge}\right\|_{L^{2}\left(\mathrm{R}^{n}\right)}
$$

(since $A$ is homogeneous and elliptic)

$$
\sim\|A u\|_{Z^{s-2}\left(\mathbf{R}^{n}\right)} \text {. }
$$

So $u \in Z^{s}\left(\mathbf{R}^{n}\right) \cap L^{2}\left(\mathbf{R}^{n}\right)$ and hence $u \in H^{s}\left(\mathbf{R}^{n}\right)$.

(b) Let $A=A_{1}+A_{2}$, where $A_{2}$ is the (homogeneous and elliptic) highest order part of $A$. We prove the theorem by induction on $s$. Firstly, the result is trivial for $s \leqslant 0$. Suppose then it is true for $s \leqslant k$, where $k \geqslant 0$, and take $k<s \leqslant k+1$. By part (a) it suffices to prove that $A_{2} u \in Z^{s-2}\left(\mathbf{R}^{n}\right)$. Now $A u \in H^{s-2}\left(\mathbf{R}^{n}\right) \subset H^{k-2}\left(\mathbf{R}^{n}\right)$ and so, by the induction hypothesis, $u \in H^{k}\left(\mathbf{R}^{n}\right)$. So $A_{1} u \in H^{k-1}\left(\mathbf{R}^{n}\right) \subset H^{s-2}\left(\mathbf{R}^{n}\right)$. Hence $A_{2} u=A u-A_{1} u \in H^{s-2}\left(\mathbf{R}^{n}\right)$. If $s \geqslant 2, A_{2} u \in Z^{s-2}\left(\mathbf{R}^{n}\right)$ as required. If $0<s<2$ then 
$A_{2} u \in H^{s-2}\left(\mathbf{R}^{n}\right) \cap Z^{-2}\left(\mathbf{R}^{n}\right)$. So $\left(1+|\xi|^{2}\right)^{(s-2) / 2}\left(A_{2} u\right)^{\wedge}$ and $|\xi|^{-2}\left(A_{2} u\right)^{\wedge}$ are both $L^{2}$ functions. It follows readily that $|\xi|^{s-2}\left(A_{2} u\right)^{\wedge} \in L^{2}\left(\mathbf{R}^{n}\right)$ and so $A_{2} u \in Z^{s-2}\left(\mathbf{R}^{n}\right)$.

THEOREM 5.2. Let $A$ be homogeneous and properly elliptic with $s \neq \frac{1}{2},-\frac{1}{2},-\frac{3}{2}, \ldots$. If $u \in L^{2}\left(\mathbf{R}_{+}^{n}\right)$ with $A u=0$ and $\gamma u \in Z^{s-\frac{1}{2}}\left(\mathbf{R}^{n-1}\right)$ then $u \in H^{s}\left(\mathbf{R}_{+}^{n}\right)$.

Proof. Let $g=\gamma u$ and, in the notation of the proof of Theorem 2.1, let $(Q g)^{\wedge}(\xi)=h\left(\xi^{\prime}\right)\left|\xi^{\prime}\right|^{m}\left(\xi_{n}+i\left|\xi^{\prime}\right|\right)^{1-m} A(\xi)^{-1} \hat{g}\left(\xi^{\prime}\right)$. Since

$$
g \in Z^{-\frac{1}{2}}\left(\mathbf{R}^{n-1}\right), \quad Q g \in L^{2}\left(\mathbf{R}^{n}\right) .
$$

But also $g \in Z^{s-\frac{1}{1}}\left(\mathbf{R}^{n-1}\right)$ and so $Q g \in Z^{s}\left(\mathbf{R}^{n}\right)$. Therefore $Q g \in H^{s}\left(\mathbf{R}^{n}\right)$. But $R Q g=u$, as proved before. So $u \in H^{s}\left(\mathbf{R}_{+}^{n}\right)$.

THEOREM 5.3. Let $A$ be properly elliptic with $s$ real and $r>\frac{1}{2}$.

(a) If $A$ is homogeneous, with $u \in H^{r}\left(\mathbf{R}_{+}^{n}\right), A u \in Z^{s-2}\left(\mathbf{R}_{+}^{n}\right)$ and $\gamma u \in Z^{s-1}\left(\mathbf{R}^{n-1}\right)$, then $u \in H^{s}\left(\mathbf{R}_{+}^{n}\right)$.

(b) In general, if $u \in H^{r}\left(\mathbf{R}_{+}^{n}\right), A u \in H^{s-2}\left(\mathbf{R}_{+}^{n}\right)$ and $\gamma u \in H^{s-1}\left(\mathbf{R}^{n-1}\right)$, then $u \in H^{s}\left(\mathbf{R}_{+}^{n}\right)$.

Proof. (a) If $s<r$ there is nothing to prove, so take $s \geqslant r$. Then $s>\frac{1}{2}$ and by Corollary 2.3 the operator $(A, \gamma): Z^{s}\left(\mathbf{R}_{+}^{n}\right) \rightarrow Z^{s-2}\left(\mathbf{R}_{+}^{n}\right) \times Z^{s-\frac{1}{1}}\left(\mathbf{R}^{n-1}\right)$ is an isomorphism. We construct an inverse $G=G_{s}$ of $(A, \gamma)$ as follows.

First, recall that $\gamma / \operatorname{ker} A$ is an isomorphism with inverse $E=R Q$. Next, let $P: Z^{s-2}\left(\mathbf{R}_{+}^{n}\right) \rightarrow Z^{s-2}\left(\mathbf{R}^{n}\right)$ be the reflection operator constructed in Pryde (1979b), Section 4. In particular, $R P=I$. (If $s-2<0, P$ and $R$ were denoted $j^{*}$ and $i^{*}$ respectively.) By Corollary 1.4, $A: Z^{s}\left(\mathbf{R}_{+}^{n}\right) \rightarrow Z^{s-2}\left(\mathbf{R}_{+}^{n}\right)$ is right invertible, and, in fact, a right inverse is $F=R A^{-1} P$. Take

$$
G=[(I-E \gamma) F, E]=R\left[(I-Q \gamma) A^{-1} P, Q\right] .
$$

Then

$$
\left[\begin{array}{l}
A \\
\gamma
\end{array}\right] G=\left[\begin{array}{ll}
A(I-E \gamma) F & A E \\
\gamma(I-E \gamma) F & \gamma E
\end{array}\right]=\left[\begin{array}{ll}
I & 0 \\
0 & I
\end{array}\right]
$$

and

$$
\begin{aligned}
G\left[\begin{array}{c}
A \\
\gamma
\end{array}\right] & =(I-E \gamma) F A+E \gamma \\
& =(I-E \gamma)(I-(I-F A))+E \gamma \\
& =I-(I-E \gamma)(I-F A) \\
& =I
\end{aligned}
$$

because $I-F A$ maps into $\operatorname{ker} A$. 
Now let $u \in H^{r}\left(\mathbf{R}_{+}^{n}\right)$ with $A u \in Z^{s-2}\left(\mathbf{R}_{+}^{n}\right)$ and $\gamma u \in Z^{s-\frac{1}{2}}\left(\mathbf{R}^{n-1}\right)$. Set $w=(A u, \gamma u)$. Then $u=G_{r} w$. Since $u \in H^{r}\left(\mathbf{R}_{+}^{n}\right),(A u)^{\wedge}$ and $(\gamma u)^{\wedge}$ are functions, and so therefore is $(G w)^{\wedge}$. Hence

$$
G_{r} w=|\nabla|-r\left(|\xi| r(G w)^{\wedge}\right)^{\curvearrowright}
$$

and

$$
G_{s} w=|\nabla|^{-s}\left(|\xi|^{s}(G w)^{\wedge}\right)^{`}
$$

It follows that $u=G_{r} w=G_{s} w \in Z^{s}\left(\mathbf{R}_{+}^{n}\right)$. But $u \in H^{r}\left(\mathbf{R}_{+}^{n}\right)$ and so $u \in H^{s}\left(\mathbf{R}_{+}^{n}\right)$ as required.

(b) Let $A=A_{1}+A_{2}$ as before. Again we prove the theorem by induction on $s$, the result being trivial for $s \leqslant r$. Suppose then it is true for $s \leqslant r+k$ where $k \geqslant 0$ and take $r+k<s \leqslant r+k+1$. By the induction hypothesis, $u \in H^{r+k}\left(\mathbf{R}_{+}^{n}\right)$. So

and therefore

$$
A_{1} u \in H^{r+k-1}\left(\mathbf{R}_{+}^{n}\right) \subset H^{s-2}\left(\mathbf{R}_{+}^{n}\right)
$$

$$
A_{2} u=A u-A_{1} u \in H^{s-2}\left(\mathbf{R}_{+}^{n}\right) .
$$

If $s \geqslant 2, A_{2} u \in Z^{s-2}\left(\mathbf{R}_{+}^{n}\right)$ and the result follows from part (a). If $r<s<2$ then $P A_{2} u \in H^{s-2}\left(\mathbf{R}^{n}\right) \cap Z^{r-2}\left(\mathbf{R}^{n}\right)$. So $\left(1+|\xi|^{2}\right)^{(s-2) / 2}\left(P A_{2} u\right)^{\wedge}$ and $|\xi|^{r-2}\left(P A_{2} u\right)^{\wedge}$ are both $L^{2}$ functions. Hence $|\xi|^{s-2}\left(P A_{2} u\right)^{\wedge} \in L^{2}\left(\mathbf{R}^{n}\right)$ and $P A_{2} u \in Z^{s-2}\left(\mathbf{R}^{n}\right)$. So $A_{\mathbf{2}} u \in Z^{s-2}\left(\mathbf{R}_{+}^{n}\right)$ and the result follows from part (a).

Using the isomorphism $B^{\prime}$, when $B$ is homogeneous, or $B_{2}^{\prime}$ otherwise ( $B_{2}$ denoting the highest order part of $B$ ), we obtain from Theorem 5.2 and a simple modification of the proof of Theorem 5.3.

THEOREM 5.4. Let $A$ be homogeneous and properly elliptic, B homogeneous and satisfying the complementing condition, and $s \neq \frac{3}{2}, \frac{1}{2},-\frac{1}{2}, \ldots$ If $u \in L^{2}\left(\mathbf{R}_{+}^{n}\right)$ with $A u=0$ and $B u \in Z^{s-(3 / 2)}\left(\mathbf{R}^{n-1}\right)$ then $u \in H^{s}\left(\mathbf{R}_{+}^{n}\right)$.

THEOREM 5.5. Let $A$ be properly elliptic, $B$ satisfy the complementing condition, $s$ be real and $r>\frac{3}{2}$.

(a) If $A$ and $B$ are homogeneous, with $u \in H^{r}\left(\mathbf{R}_{+}^{n}\right), A u \in Z^{s-2}\left(\mathbf{R}_{+}^{n}\right)$ and $B u \in Z^{s-(3 / 2)}\left(\mathbf{R}^{n-1}\right)$, then $u \in H^{s}\left(\mathbf{R}_{+}^{n}\right)$.

(b) In general, if $u \in H^{r}\left(\mathbf{R}_{+}^{n}\right), A u \in H^{s-2}\left(\mathbf{R}_{+}^{n}\right)$ and $B u \in H^{8-(3 / 2)}\left(\mathbf{R}^{n-1}\right)$, then $u \in H^{s}\left(\mathbf{R}_{+}^{n}\right)$.

\section{References}

S. Agmon, A. Douglis and L. Nirenberg (1959), 'Estimates near the boundary for solutions of elliptic partial differential equations satisfying general boundary conditions, I', Comm. Pure Appl. Math. 12, 623-727. 
F. E. Browder (1959), 'Estimates and existence theorems for elliptic boundary value problems', Proc. Nat. Acad. Sci. U.S.A. 45, 365-372.

A. J. Pryde (1977), 'The five lemma for Banach spaces', Proc. Amer. Math. Soc. 65 (1), 37-43.

A. J. Pryde (1979a), 'Spaces with homogeneous norms', Bull. Austral. Math. Soc. (to appear).

A. J. Pryde (1979b), 'Second order elliptic equations with mixed boundary conditions', submitted.

M. Schechter (1959), 'Integral inequalities for partial differential operators and functions satisfying general boundary conditions', Comm. Pure Appl. Math. 12, 37-66.

School of Mathematics and Physics

Macquarie University

North Ryde, N.S.W. 2113

Australia 